\title{
INTERPOLATION OF VARIETIES OF MINIMAL DEGREE
}

\author{
AARON LANDESMAN
}

\begin{abstract}
It is well known that one can find a rational normal curve in $\mathbb{P}^{n}$ through $n+3$ general points. We prove a generalization of this to higher dimensional varieties, showing that smooth varieties of minimal degree can be interpolated through points and linear spaces.
\end{abstract}

\section{INTRODUCTION}

In this paper, we investigate the question of interpolation, which asks whether we can fit a given type of variety through a general collection of points in projective space. As one of the simplest examples of interpolation, if one chooses 5 points in $\mathbb{P}^{2}$, one can always find a degree 2 curve passing through those five points, so degree 2 curves in $\mathbb{P}^{2}$ satisfy interpolation.

Recall that a $k$ dimensional variety of degree $d$ in $\mathbb{P}^{n}$ is of minimal degree if it is nondegenerate and $d=n+1-k$. There is a simple classification of varieties of minimal degree as detailed in [EH87. Theorem 1]: If $X$ is a smooth irreducible nondegenerate variety of minimal degree in $\mathbb{P}^{n}$, then $X$ is either a quadric hypersurface, a rational normal scroll, the image of $\mathbb{P}^{2}$ under the 2-Veronese embedding $v_{2}\left(\mathbb{P}^{2}\right) \rightarrow \mathbb{P}^{5}$, or $\mathbb{P}^{n}$ itself. In this paper, we show that smooth varieties of minimal degree satisfy interpolation.

We next define more precisely what we mean by interpolation. Suppose $\mathrm{U}$ is an integral subscheme of the Hilbert scheme parameterizing varieties of dimension $\mathrm{k}$ in $\mathbb{P}^{\mathrm{n}}$. Define $\mathrm{q}$ and $\mathrm{r}$ so that $\operatorname{dim} U=q \cdot(n-k)+r$. We say $U$ satisfies interpolation if for any collection $p_{1}, \ldots, p_{q}, \Lambda$, where $p_{i} \in \mathbb{P}^{n}$ are points and $\Lambda \subset \mathbb{P}^{n}$ is a plane of dimension $n-k-r$, there exists some $[Y] \in U$ so that $Y$ passes through $p_{1}, \ldots, p_{q}$ and meets $\Lambda$. We say $U$ satisfies weak interpolation if there exists some $[\mathrm{Y}] \in \mathrm{U}$ meeting q general points $p_{1}, \ldots, p_{q}$. If $X$ is a projective variety lying on a unique irreducible

Date: September 24, 2018. 
component of the Hilbert scheme, denoted $\mathcal{H}_{X}$, then we say $\mathrm{X}$ satisfies interpolation if $\mathcal{H}_{X}$ does. Although this description of interpolation, given in [LP16b, Theorem A.7(9)], is the most classical one, there are at least twenty two equivalent descriptions of interpolation under moderate hypotheses, as described in [LP16b, Theorem A.7].

Interpolation has applications to Gromov-Witten theory, the slope conjecture, constructing degenerations, and understanding the Hilbert scheme, as detailed in [LP16b, Subsection 1.1]. Also see [LP16b, Section 1] for a more leisurely introduction to interpolation. It is known that nearly all nonspecial curves satisfy interpolation [ALY15], and that canonical curves in genus other than 6 and 4 satisfy weak interpolation as proven in [Ste03, Chapter 13]. Most recently, it was shown that smooth del Pezzo surfaces satisfy weak interpolation [LP16b, Theorem 1.1].

We now state our main result, which continues the investigation of interpolation of higher dimensional varieties. The theorem holds over an algebraically closed field of arbitrary characteristic.

Theorem 1.1. Smooth varieties of minimal degree satisfy interpolation.

As a consequence, varieties of minimal degree satisfy strong interpolation (which is a stronger notion of interpolation discussed preceding Corollary 3.14) in characteristic 0, as proven in Corollary 3.14.

Remark 1.2. Although several bits and pieces of Theorem 1.1 were previously known, the unknown cases are the trickiest ones to deal with in the proof we present.

We now describe those parts of Theorem 1.1 which were previously established. The dimension 1 case is the well known fact that one can find a rational normal curve through $n+3$ points in $\mathbb{P}^{n}$. The Veronese surface was shown to satisfy interpolation in [Cob. Theorem 19], which is summarized in a more modern treatment in [LP16b, Theorem 5.6], piecing together the ideas in [Dol04, Theorem 5.2, Theorem 5.6]. It was already established that 2-dimensional scrolls satisfy interpolation in Coskun's thesis [Cos06a, Example, p. 2], and furthermore, Coskun gives a method for computing the number of scrolls meeting a specified collection of general linear spaces. Finally, weak interpolation was established for scrolls of degree $d$ and dimension $k$ with $d \geq 2 k-1$ via the Gale transform in [EP98, Theorem 4.5] and independently via an explicit construction in [CRV95, Theorem 3.2]. Further, in the case $k \leq d<2 k-1$, it was shown in [CRV95, Theorem 3.2] that scrolls pass through many points, though the resulting number is one point short of establishing weak interpolation. 
While our methods are similar to those of [Cos06a], they differ drastically from those of [EP98, Theorem 4.5] and [CRV95, Theorem 3.2].

1.1. Notations and Conventions. We work over an algebraically closed field $\mathbb{k}$ of arbitrary characteristic. We notate a sequence $(a, \ldots, a)$ of length $b$ as $\left(a^{b}\right)$. So, for example, we would notate $(1,1,1,2,3,3)$ as $\left(1^{3}, 2,3^{2}\right)$. We also include the following idiosyncratic notation, mostly regarding scrolls:

(1) When $X$ lies on a unique irreducible component of the Hilbert scheme, we let $\mathcal{H}_{X}$ denote that irreducible component and let $V_{X}$ denote the universal family over that component.

(2) Recall that a scroll is a projective bundle $\pi: X \rightarrow \mathbb{P}^{1}$ embedded into projective space by $\mathcal{O}_{\pi}(1)$. If $X \cong \mathbb{P} \mathcal{E}$ with $\mathcal{E} \cong$ $\mathcal{O}\left(a_{1}\right) \oplus \cdots \oplus\left(a_{k}\right)$, we say $X$ is of type $\left(a_{1}, \ldots, a_{k}\right)$. We use $S_{a_{1}, \ldots, a_{k}}$ to refer to a smooth scroll of type $\left(a_{1}, \ldots, a_{k}\right)$.

(3) When dealing with a scroll $X$ in projective space, we use $d$ to refer to its degree, $k$ to refer to its dimension, and $n:=$ $\mathrm{d}+\mathrm{k}-1$ to refer to the dimension of the ambient projective space, $X \subset \mathbb{P}^{n}$.

(4) If $X$ is a smooth scroll of degree $d$ and dimension $k$, we use $\mathcal{H}_{\mathrm{d}, \mathrm{k}}^{\text {scroll }}:=\mathcal{H}_{X}$.

(5) Let $X$ be a smooth scroll of degree $d$ and dimension $k$. We use $\mathcal{H}_{\mathrm{d}, \mathrm{k}, \mathrm{sm}}^{\mathrm{scroll}}$ to refer to the complement in $\mathcal{H}_{\mathrm{X}}$ of the image under $\pi: \mathcal{V}_{X} \rightarrow \mathcal{H}_{X}$ of the singular locus of the map $\pi: \mathcal{V}_{X} \rightarrow \mathcal{H}_{X}$.

(6) We use $\mathcal{H}_{d, k}^{\text {broken }}$ to denote the closure of the locus of points in $\mathcal{H}_{d, k}^{\text {scroll }}$ corresponding to varieties which are the union of a $k$-plane and a degree $d-1$, dimension $k$ scroll, intersecting in a $(k-1)$-plane which is a ruling plane of the degree $d-1$ scroll.

1.2. Acknowledgements. I thank Joe Harris and Anand Patel for advising this project. For helpful conversations and correspondence, I thank Atanas Atanasov, Francesco Cavazzani, Brian Conrad, Izzet Coskun, Anand Deopurkar, Phillip Engel, Changho Han, Joe Harris, Brendan Hassett, Allen Knutsen, Eric Larson, John Lesieutre, Rahul Pandharipande, Anand Patel, Alex Perry, Geoffrey Smith, Hunter Spink, Ravi Vakil, Adrian Zahariuc, Yifei Zhao, Yihang Zhu, and David Zureick-Brown. 


\section{BACKGROUND ON SCROLLS}

In this section, we collect background results on several descriptions of the structure of scrolls, which we later use to show scrolls satisfy interpolation. In Lemma 2.1 we show certain Segre embeddings are scrolls, in Proposition 2.2, we describe Fano schemes of scrolls, in Proposition 2.3. we show smooth varieties of minimal degree correspond to smooth points of the Hilbert scheme, in Lemma 2.5 we describe possible degenerations of scrolls, and in Lemma 2.6. we describe one method of constructing certain scrolls.

Lemma 2.1. The scroll $S_{1^{k}}$ of dimension $\mathrm{k}$ in $\mathbb{P}^{2 \mathrm{k}-1}$ is, up to automorphism of $\mathbb{P}^{2 \mathrm{k}-1}$, isomorphic to the Segre embedding $\mathbb{P}^{1} \times \mathbb{P}^{\mathrm{k}-1} \rightarrow \mathbb{P}^{2 \mathrm{k}-1}$.

Proof. Observe that by definition we have $S_{1^{k}} \cong \mathbb{P}\left(\oplus_{i=1}^{k} \mathcal{O}_{\mathbb{P}^{1}}(1)\right)$. So, abstractly, $S_{1^{k}}$ is the trivial bundle over $\mathbb{P}^{1}$, isomorphic to $\mathbb{P}^{1} \times \mathbb{P}^{k-1}$. Further, the invertible sheaf $\mathcal{O}_{\pi}(1)$ indeed embeds $S_{1 k}$ via the Segre embedding $\mathbb{P}^{1} \times \mathbb{P}^{k-1} \rightarrow \mathbb{P}^{2 k-1}$.

Proposition 2.2. Suppose $\mathrm{X} \subset \mathbb{P}^{n}$ is a smooth scroll of minimal degree $\mathrm{d}$ and dimension $\mathrm{k}$, where $\pi: \mathrm{X} \cong \mathbb{P} \mathcal{E} \rightarrow \mathbb{P}^{1}$ is the projection, for $\mathcal{E}$ a locally free sheaf on $\mathbb{P}^{1}$. Further, let $\mathrm{X} \cong S_{a_{1}, \ldots, a_{s}, 1 j}$ with $a_{1} \geq \cdots \geq a_{s}>1$. Suppose that $\mathrm{X} \subset \mathbb{P}^{\mathrm{n}}$ is embedded so that it is the span of the planes joining rational normal curves $\mathrm{C}_{1}, \ldots, \mathrm{C}_{s}, \mathrm{~L}_{1}, \ldots, \mathrm{L}_{\mathrm{j}}$ where $\mathrm{L}_{1}, \ldots, \mathrm{L}_{\mathrm{j}}$ are lines and $\mathrm{C}_{1}, \ldots, \mathrm{C}_{\mathrm{s}}$ are rational normal curves of degree at least 2 . Let $\mathrm{t}$ be an integer with $1 \leq \mathrm{t} \leq \mathrm{k}-1$. Then, the $\mathrm{t}$-planes contained in $\mathrm{X}$ are of one of the following two forms:

1 If $\mathrm{j}>1$, let $\mathrm{P} \subset \mathbb{P V}$ be written as $\mathrm{P}=\mathbb{P W}$ for a two dimensional subspace $\mathrm{W} \subset \mathrm{V}$, and let $\mathrm{L}_{1}, \ldots, \mathrm{L}_{\mathrm{j}}$ be the projectivizations of planes $\mathrm{P}_{1}, \ldots, \mathrm{P}_{\mathrm{j}} \subset \mathrm{V}$. Then, $\mathrm{W} \in \operatorname{Span}\left(\mathrm{P}_{1}, \ldots, \mathrm{P}_{\mathrm{j}}\right)$.

$2 \mathrm{P}$ is contained in some $(\mathrm{k}-1)$-plane which is the fiber of the projection map $\pi: \mathrm{X} \rightarrow \mathbb{P}^{1}$.

The Fano scheme of $\mathrm{t}$-planes in $\mathrm{X}$ is smooth. If $\mathrm{j} \geq 1$ and $\mathrm{t}=1$, it has two irreducible components, corresponding to planes of type 1 and 2 It has one irreducible component corresponding to planes of type 2 otherwise. The component of planes of type 2 is isomorphic to the Grassmannian bundle $\mathbb{G}(t+1, \mathcal{E})$ over $\mathbb{P}^{1}$. If it exists, meaning $t=1$ and $j \geq 1$, the component of planes of type 1 is isomorphic to $\mathbb{P}^{\mathbf{j}-1}$.

One fairly routine proof follows by first describing the Fano scheme first set theoretically, then scheme theoretically, and then verifying cohomologically that planes are smooth points of the Fano scheme. See [LP16a, Proposition 5.2.2] for a detailed proof. 
Proposition 2.3. If $X$ is a smooth variety of minimal degree then $\mathrm{H}^{1}\left(\mathrm{X}, \mathrm{N}_{X / \mathbb{P}^{n}}\right)=$ 0 . In particular, such a variety is a smooth point of the Hilbert scheme. Further, if $\mathrm{X}$ is a smooth scroll of minimal degree $\mathrm{d}$ and dimension $\mathrm{k}$ in $\mathbb{P}^{\mathrm{n}}$ then $\mathrm{h}^{0}\left(\mathrm{X}, \mathrm{N}_{\mathrm{X} / \mathbb{P}^{\mathrm{n}}}\right)=(\mathrm{d}+\mathrm{k})^{2}-\mathrm{k}^{2}-3$. In particular, $\operatorname{dim} \mathcal{H}_{\mathrm{d}, \mathrm{k}}^{\text {scroll }}=$ $(d+k)^{2}-k^{2}-3$.

See [LP16a, Proposition 5.3.2, Proposition 5.3.5 and Proposition 5.3.16] for a more detailed proof than the sketch given below.

Proof. First, by [EH87, Theorem 1], we only need verify this when $\mathrm{X}$ is a quadric surface, the 2 Veronese, or a smooth scroll. The case of hypersurfaces follows since for $X \subset \mathbb{P}^{n}$ a hypersurface, $N_{X / \mathbb{P}^{n}} \cong$ $\mathcal{O}(\operatorname{deg} X)$. The case of the 2 -Veronese follows from a fairly straightforward chase of long exact sequences using the normal exact sequence and Euler exact sequence. Finally, the case of scrolls is somewhat more involved, but follows from chasing exact sequences using the normal exact sequence, the relative tangent exact sequence (see [Bra14, Theorem 4.5.13]), the Euler exact sequence, and the Leray spectral sequence.

Remark 2.4. For the remainder of the paper, instead of working in the compactification of the locus of smooth scrolls given by the Hilbert scheme, we work in the compactification of smooth scrolls of dimension $k$ and degree $d$ given by rational curves of degree $d$ in the Grassmannian $G(k, k+d)$. This is described in the case $k=2$ in [Cos06a, Section 3], and the generalization to $k>2$ is completely analogous.

Since the Hilbert scheme and the compactification of rational curves in this Grassmannian are birational, the number of scrolls through a general collection of planes of specified dimensions is the same for either compactification. So to prove interpolation holds, it suffices to show there is a point in this compactification of rational curves corresponding to a scroll through a general collection of linear spaces.

The following is a direct generalization of [Cos06b, Proposition $4.1]$, and the proof is nearly the same, mutatis mutandis.

Lemma 2.5 (Generalization of [Cos06a, Proposition 4.1]). Suppose $X \rightarrow$ $\mathrm{B}$ is a flat family with B a curve so that the general fiber is a smooth scroll, and that the special fiber is nondegenerate. Then, the special fiber is a connected variety whose irreducible components are scrolls.

Lemma 2.6. Fix $k$ lines $\ell_{1}, \ldots, \ell_{k} \subset \mathbb{P}^{2 k-1}$ and three $(k-1)$-planes $\Lambda_{1}, \Lambda_{2}, \Lambda_{3} \subset \mathbb{P}^{2 k-1}$. Suppose that $\ell_{i} \cap \Lambda_{j}$ consists of a single point for all pairs $(i, j)$, and that no two of these intersection points are the same. 
Further, suppose the lines $\ell_{1}, \ldots, \ell_{k}$ span $\mathbb{P}^{2 \mathrm{k}-1}$. Then, there is a unique scheme $\mathrm{X} \subset \mathbb{P}^{2 \mathrm{k}-1}$ so that $\mathrm{X} \cong \mathbb{P}^{1} \times \mathbb{P}^{k-1}$ is the Segre embedding and $\mathrm{X}$ contains $\ell_{1}, \ldots, \ell_{k}, \wedge_{1}, \wedge_{2}, \wedge_{3}$.

Further, the lines $\ell_{1}, \ldots, \ell_{k}$ appear as the images of lines of the form $\mathbb{P}^{1} \times\left\{q_{k}\right\} \subset \mathbb{P}^{1} \times \mathbb{P}^{k-1}$ under the Segre embedding, with $\mathrm{q}_{i} \in \mathbb{P}^{\mathrm{k}-1}$.

For a more detailed proof, see [LP16a, Lemma 7.9.1].

Proof. This is a special case of the fact that a scroll is uniquely determined as the planes swept out by a collection of rational normal curves with compatible isomorphisms.

\section{INTERPOLATION OF VARIETIES OF MINIMAL DEGREE}

In this section, we prove that any smooth variety of minimal degree satisfies interpolation. We work not in the compactification of smooth scrolls given by the Hilbert scheme, but rather that given by rational curves in the Grassmannian, as described in Remark 2.4. By [EH87, Theorem 1], we can show this separately in the cases of a Hilbert scheme whose general member is a quadric hypersurface, the Veronese surface in $\mathbb{P}^{5}$ and a smooth rational normal scroll. Since quadrics satisfy interpolation and the 2-Veronese satisfies interpolation by [LP16b, Theorem 5.6], we shall concentrate on showing that rational normal scrolls satisfy interpolation. The general idea of the proof is to fix the dimension of scrolls, and induct on the degree. The elements of this inductive argument is summarized verbally in Theorem 3.13 and pictorially in Figure 7. Most of the remaining subsections each prove one of the Propositions appearing in Figure 7.

3.1. The set-up for scrolls. In order to show scrolls satisfy interpolation, we start by stating the precise number of points and linear spaces a scroll must pass through to satisfy interpolation.

Lemma 3.1. The condition of interpolation means that we can find a degree $\mathrm{d}$ dimension $\mathrm{k}$ scroll

- containing $\mathrm{d}+2 \mathrm{k}+1$ general points and meeting a general $\mathrm{d}-$ $2 \mathrm{k}+1$-plane if $\mathrm{d} \geq 2 \mathrm{k}-1$,

- containing $\mathrm{d}+2 \mathrm{k}+2$ general points and meeting a general $2(\mathrm{~d}-$ $\mathrm{k}$ )-plane if $\mathrm{k} \leq \mathrm{d} \leq 2 \mathrm{k}-2$.

Proof. By Proposition 2.3, we know

$$
\begin{aligned}
\operatorname{dim} \mathcal{H}_{d, k}^{\text {scroll }} & =(d+k)^{2}-k^{2}-3 \\
& =(d-1)(d+2 k+1)+2 k-2 .
\end{aligned}
$$


Note that we always have $d \geq k$ and $2 k-2 \leq d-1$ when $d \geq$ $2 k-1$. Therefore, in the case $d>2 k-1$, interpolation means the scroll passes through $d+2 k+1$ points and meets a $(d-2 k+1)-$ plane. Next, when $k<d \leq 2 k-2$, we have $d-1<2 k-2<$ $2(d-1)$, and so for interpolation to hold, such schemes must pass through $d+2 k+2$ points and meet a $2(d-k)$-plane.

We prove that scrolls satisfy interpolation by induction. We fix an integer $k$ and induct on the degree of $k$-dimensional varieties. In order to prove the theorem for a variety of dimension $k$ and degree $\mathrm{d}$, we make the following inductive hypotheses.

ind-1 When $d>k$, we assume $\mathcal{H}_{d-1, k}^{\text {scroll }}$ satisfies interpolation.

ind-2 When $k<d \leq 2 k-1$, we assume there is a variety in $\mathcal{H}_{d-1, k}^{\text {scroll }}$ containing a general $(2 k-d-1)$-plane and containing $2 d$ points.

3.2. Inductive degeneration for degree at least $2 k-1$. The main aim of this subsection is to prove Proposition 3.2, showing that degree $d$ scrolls satisfy interpolation for $d \geq 2 k-1$, assuming our inductive hypothesis ind-1.

Proposition 3.2. Assuming induction hypothesis ind-1, if $\mathrm{d}>2 \mathrm{k}-1$ then $\mathcal{H}_{\mathrm{d}, \mathrm{k}}^{\text {scroll }}$ satisfies interpolation.

Remark 3.3. Before proving Proposition 3.2, let us note that it immediately gives another proof that there is a rational normal curve through $n+3$ points in $\mathbb{P}^{n}$. The proof holds by induction on the degree, which covers all cases when $k=1$ because when $k=1$, $2 \mathrm{k}-1=1$. This is pictorially summarized in Figure 2 .

The idea of the proof of Proposition 3.2 is to specialize all but two of the points to a hyperplane, and then find a reducible scroll of degree $d$ which is the union of a scroll of degree $d-1$ in a hyperplane and $\mathbb{P}^{k}$, meeting along $\mathbb{P}^{k-1}$, as pictured in Figure 1

Proof. By Lemma 3.1, $\mathcal{H}_{d, k}^{\text {scroll }}$ satisfying interpolation means that given a collection of $d+2 k+1$ points and a general $(d-2 k+1)$-plane, $\Lambda$, we can find some $[Z] \in \mathcal{H}_{d, k}^{\text {scroll }}$, so that $Z$ passes through the points and meets $\Lambda$. Now specialize $d+2 k-1$ of the points $p_{1}, \ldots, p_{d+2 k-1}$ to a general hyperplane $H \subset \mathbb{P}^{d+k-1}$. Let $\ell$ be the line through $p_{d+2 k}, p_{d+2 k+1}$ and let $q:=\ell \cap H$. By induction hypothesis ind-1, there is a degree $d-1$ dimension $k$ variety of minimal degree which contains the $(d-1)+2 k+1$ points $p_{1}, \ldots, p_{d+2 k-1}, q$ and meets the $((d-1)-2 k+1)$-plane $H \cap \wedge$. Call that variety $X$. Since $q \in X$, there is a unique $(k-1)$-plane contained in $X$ and containing $q$, as 


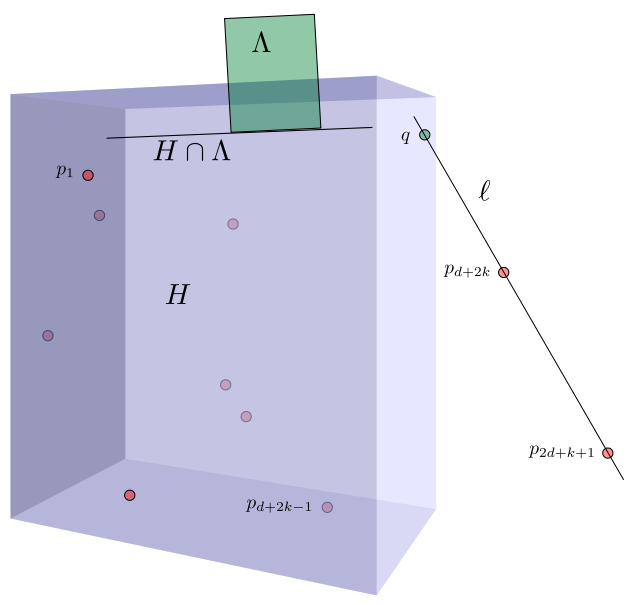

FIGURE 1. A visualization of the idea of the proof of Proposition 3.2, where one inductively specializes all but two points to lie in a hyperplane.
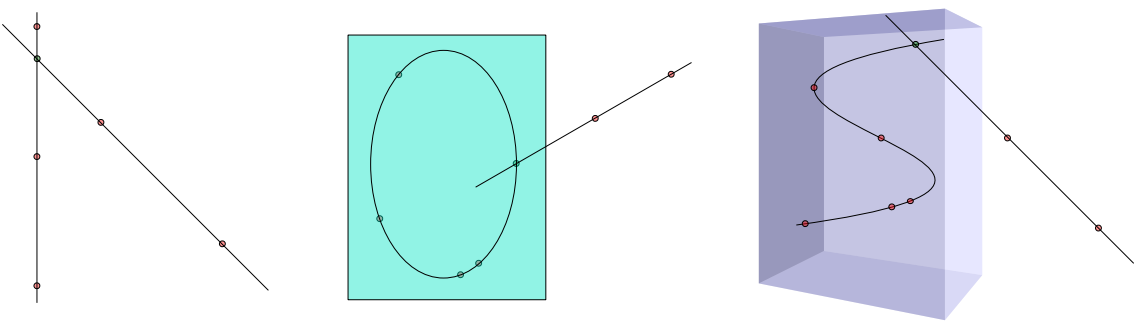

FIGURE 2. A pictorial description of the inductive degeneration following Proposition 3.2 for showing rational normal curves satisfy interpolation, see also $\mathrm{Re}-$ mark 3.3. Degenerations are drawn for rational normal curves of degrees 2,3 , and 4 .

follows from Proposition 2.2. Let that $k-1$-plane be $P$ and let $Y$ be the $k$-plane spanned by $\ell$ and $P$. Then, the variety $X \cup Y$ with reduced scheme structure lies in $\mathcal{H}_{\mathrm{d}, \mathrm{k}}^{\text {scroll }}$ by Lemma 2.5.

In order to show $\mathcal{H}_{d, k}^{\text {scroll }}$ satisfies interpolation, it suffices to show $X \cup Y$ is an isolated point in the set of all elements of $\mathcal{H}_{\mathrm{d}, \mathrm{k}}^{\text {scroll }}$ containing $p_{2}, \ldots, p_{d+2 k-1}$ and $\Lambda$, by [LP16b, Thorem A.7(5)]. Since the points and plane were chosen generally subject to the requirement that $\mathrm{k}+\mathrm{d}$ of the points were contained in a hyperplane, by Lemma 2.5 , it suffices to show there are only finitely many scrolls in $\mathcal{H}_{\mathrm{d}, \mathrm{k}}^{\text {broken }} \cup$ 
$\mathcal{H}_{d, k, s m}^{\text {scroll }}$ (recall these were defined in subsection 1.1 containing $p_{1}, \ldots, p_{d-2 k+1}$, and meeting $\Lambda$.

This now follows from a dimension count. First, we show there are only finitely many scrolls in $\mathcal{H}_{d, k}^{\text {broken }}$ containing $p_{1}, \ldots, p_{d-2 k+1}$ and meeting $\Lambda$. Because of our specialization of the points, any $[X \cup Y] \in$ $\mathcal{H}_{\mathrm{d}, \mathrm{k}}^{\text {broken }}$ with $\mathrm{Y} \cong \mathbb{P}^{k-1}$ containing this set of points must satisfy $\mathrm{X} \subset$ $H$. By our induction hypothesis, there are a positive finite number of scrolls $X$ in $H$ meeting $p_{1}, \ldots, p_{d-2 k-1}, q$, and $\wedge \cap H$. Once $X$ is chosen, $\mathrm{Y}$ is uniquely determined, so there are finitely many choices for $X \cup Y$.

To complete the proof, it suffices to show there are only finitely many smooth scrolls containing $p_{1}, \ldots, p_{k+d+1}$ and meeting $\wedge$. This follows from Lemma 3.4, which we prove next.

Lemma 3.4. Let $\mathrm{p}_{1}, \ldots, \mathrm{p}_{\mathrm{d}+2 \mathrm{k}+1}$ be points in $\mathbb{P}^{\mathrm{n}}$ so that $\mathrm{p}_{1}, \ldots, \mathrm{p}_{\mathrm{d}+2 \mathrm{k}-1}$ are contained in a hyperplane $\mathrm{H} \subset \mathbb{P}^{n}$, but the points are otherwise general, and let $\Lambda$ be a general $(\mathrm{d}-2 \mathrm{k}+1)$ plane. Then, there are only finitely many smooth scrolls containing $p_{1}, \ldots, p_{\mathrm{d}+2 k+1}$ and meeting $\Lambda$.

Remark 3.5. Although the following proof is a fairly standard dimension counting argument, we include it for completeness. Throughout the remainder of the paper, many similar dimension counts will be done, the formal details of which are omitted as they are similar in nature to this one.

Proof. For the remainder of the proof, fix a hyperplane $\mathrm{H} \subset \mathbb{P}^{n}$. Define the incidence correspondence

$$
\begin{aligned}
\Phi:=\left(\mathcal{V}_{\mathrm{X}} \times{ }_{\mathcal{H}_{p_{1}}} \mathrm{H}\right) & \times_{\mathcal{H}_{X}} \cdots \times_{\mathcal{H}_{X}}\left(\mathcal{V}_{\mathrm{X}} \times \times_{\mathcal{H}_{p_{\mathrm{d}+2 k-1}}} \mathrm{H}\right) \\
& \times_{\mathcal{H}_{X}} \mathcal{V}_{\mathrm{X}} \times{ }_{\mathcal{H}_{X}} \mathcal{V}_{\mathrm{X}} \times{ }_{\mathcal{H}_{X}}\left(\mathcal{V}_{\mathrm{X}} \times \mathbb{P}^{n} \mathcal{H}_{\Lambda}\right)
\end{aligned}
$$

To complete the proof, it suffices to show that

$$
\operatorname{dim} \Phi=\operatorname{dim} H^{d+2 k-1} \times\left(\mathbb{P}^{n}\right)^{2} \times G(d-2 k+2, n+1) .
$$

because then the projection map

$$
\Phi \rightarrow\left(\mathbb{P}^{n}\right)^{d+2 k-1} \times\left(\mathbb{P}^{n}\right)^{2} \times G(d-2 k+2, n+1)
$$

is either generically finite or not dominant, and in either case, there are only finitely many smooth varieties through a general set of such points, meeting a general plane $\Lambda$. 
Note that

$$
\begin{aligned}
\operatorname{dim} \mathcal{V}_{X} \times_{\mathbb{P}^{n}} \mathrm{H}-\operatorname{dim} \mathcal{H}_{\mathrm{d}, \mathrm{k}}^{\text {scroll }}=\mathrm{k}-1 \\
\operatorname{dim} \mathcal{V}_{X}-\operatorname{dim} \mathcal{H}_{\mathrm{d}, \mathrm{k}}^{\mathrm{scroll}}=\mathrm{k} \\
\operatorname{dim} \mathcal{V}_{\mathrm{X}} \times_{\mathbb{P}^{n}} \mathcal{H}_{\Lambda}-\operatorname{dim} \mathcal{H}_{\mathrm{d}, \mathrm{k}}^{\text {scroll }}=\mathrm{k}+(\mathrm{d}-2 \mathrm{k}+1)(3 \mathrm{k}-2)
\end{aligned}
$$

This completes the proof because

$$
\begin{aligned}
\operatorname{dim} \Phi= & \left((d+k)^{2}-k^{2}-3\right) \\
& \quad+((k-1)(d+2 k-1)+(2 k)+(k+(d-2 k+1)(3 k-2))) \\
= & (d+k-2)(d+2 k-1)+2(d+k-1) \\
& \quad+(d-2 k+2)(n+2 k-d-1) \\
= & \operatorname{dim}\left(\mathbb{P}^{n}\right)^{d+2 k-1} \times\left(\mathbb{P}^{n}\right)^{2} \times G(d-2 k+2, n+1) .
\end{aligned}
$$

Remark 3.6. To prove Lemma 3.4, one can also do the following dimension count, although this takes a little justification to rigorize: The condition that $X$ pass through a point imposes $n-k=d-1$ conditions. When we specialize a point to a hyperplane, the hyperplane intersects $X$ in a scroll of dimension $k-1$ in $\mathbb{P}^{n-1}$. Hence, a point still imposes $n-1-(k-1)=n-k$ conditions. Finally, the condition to meet a $(d-2 k+1)$-plane imposes $n-k-(d-2 k+1)$ conditions. Adding these up, we obtain a total of

$$
\begin{aligned}
(d+2 k+1)(n-k)+n-k-(d-2 k+1) & =(k+d)^{2}-k^{2}-3 \\
& =\operatorname{dim} \mathcal{H}_{d, k}^{\text {scroll }}
\end{aligned}
$$

conditions, so there are only finitely many elements of $\mathcal{H}_{\mathrm{d}, \mathrm{k}}^{\text {scroll }}$ satisfying these conditions.

3.3. Inductive degeneration for degree between $k+1$ and $2 k-1$. We next show Proposition 3.7, which lets us verify scrolls of degree between $k+1$ and $2 k-1$ satisfy interpolation, assuming ind-2 inductively.

Proposition 3.7. Let $\mathrm{k}+1 \leq \mathrm{d} \leq 2 \mathrm{k}-1$. Assuming induction hypothesis ind-2 holds for varieties of degree $\mathrm{d}-1$ then $\mathcal{H}_{\mathrm{d}, \mathrm{k}}^{\text {scroll }}$ satisfies interpolation.

Proof. By Lemma 3.1, we would like to show there is a degree $d-1$, dimension $k$ variety passing through $d+2 k+2$ points, $p_{1}, \ldots, p_{d+2 k+2}$, and meeting a general $2(\mathrm{~d}-\mathrm{k})$ plane $\Lambda$. Choose a general hyperplane $\mathrm{H}$ and specialize $2 \mathrm{~d}$ of these points, $\mathrm{p}_{1}, \ldots, \mathrm{p}_{2 \mathrm{~d}}$, to $\mathrm{H}$. Now, 


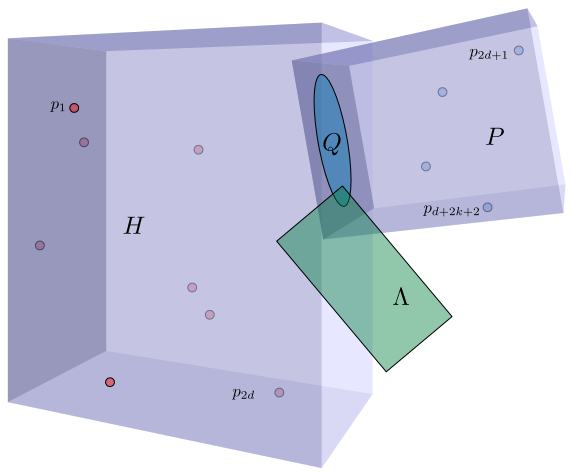

FIGURE 3. A visualization of the idea of the proof of Proposition 3.7, where one inductively specializes $2 \mathrm{~d}$ points to lie in a hyperplane $\mathrm{H}$, and the additional plane $\Lambda$ to meet the intersection of $\mathrm{H}$ and the span of the remaining $2 k-d+2$ points.

the last $2 k-d+2$ points remain general, and span some $2 k-d+1$ plane $P$. Specialize $\Lambda$ so that $\wedge \cap H \cap P \neq \varnothing$, but so that $\Lambda$ is otherwise general. Let $\mathrm{Q}:=\mathrm{H} \cap \mathrm{P}$.

Then, by induction hypothesis ind-2 for varieties of degree $d$, there is a variety of degree $d-1$ containing the $2 d$ points $p_{1}, \ldots, p_{2 d}$ and containing Q. Call this variety X.

We now explain in detail why the variety $X$ can be taken to be smooth if $p_{1}, \ldots, p_{2 d}, Q$ are chosen generally. Let $W$ be some scroll of degree $d-1$ and dimension $k$, so that $\mathcal{H}_{W}=\mathcal{H}_{d-1, k}^{\text {scroll }}$, and let $F$ denote the relative Hilbert scheme of $(2 k-d)$-planes in $\mathcal{V}_{W}$ over $\mathcal{H}_{W}$. Define

$$
\Phi:=\mathrm{F} \times \times_{\mathcal{H}_{W}} \mathcal{V}_{W} \times \mathcal{H}_{W} \cdots \times{\mathcal{H}_{W}} \mathcal{V}_{W},
$$

where there are $2 d$ copies of $V_{W}$ in the fiber product. Note that the closed points of $\Phi$ correspond to a scroll together with a $(2 k-d)-$ plane contained in it and $2 d$ points on it. Then, we have natural projection maps

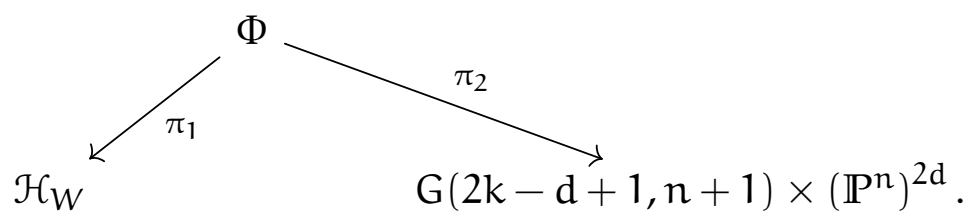

The assumption that through a general set of $2 \mathrm{~d}$ points and a general $(2 k-d)$-plane $Q$ there passes a member of $\mathcal{H}_{W}$ is equivalent to 
the map $\pi_{2}$ being dominant. Now, since the locus of smooth scrolls $\mathcal{H}_{\mathrm{d}-1, \mathrm{k}, \mathrm{sm}}^{\text {scroll }} \subset \mathcal{H}_{\mathrm{W}}$ is dense, it follows that $\pi_{1}^{-1}\left(\mathcal{H}_{\mathrm{d}-1, \mathrm{k}, \mathrm{sm}}^{\text {scroll }}\right) \subset \Phi$ is also dense, and therefore $\left.\pi_{2}\right|_{\pi_{1}^{-1}\left(\mathcal{H}_{\mathrm{d}-1, \mathrm{k}, \mathrm{sm}}^{\text {scroll }}\right)}$ is also dominant. Dominance is equivalent to the statement that for a general set of $2 \mathrm{~d}$ points and a $2 \mathrm{k}-\mathrm{d}$ plane, there is some smooth scroll passing through the points and containing the plane, as we wanted to show.

Further, if the points are chosen generally, there is some $(k-1)$ plane $R$ with $Q \subset R \subset X$, as we now explain. Note, by Proposition 2.2, if $\operatorname{dim} Q \neq 1$, then we have that $Q$ is contained in some $(k-1)$-plane. If, instead $\operatorname{dim} Q=1$, then we know there are at most two components of the Fano scheme of lines contained in $X$, and one of these has dimension $k$, which is strictly larger than the other component (if it exists), from Proposition 2.2. Therefore, since $p_{1}, \ldots, p_{d+2 k+2}$ and $\Lambda$ are chosen generally, we may assume that this line $\mathrm{Q}$ lies in the component of the Fano scheme of larger dimension. In this case, the line $Q$ lies in some $(k-1)$-plane, again by Proposition 2.2.

Define $Y$ to be the plane spanned by $R, p_{2 d+1}, \ldots, p_{d+2 k+2}$. Then, we then obtain that $X \cup Y$ corresponds to a point in $\mathcal{H}_{d, k}^{\text {scroll }}$ by Lemma 2.5.

Using dimension counts similar to, but more involved than Lemma 3.4 there are only finitely many such smooth scrolls, and finitely many such scrolls in $\mathcal{H}_{\mathrm{d}, \mathrm{k}}^{\text {broken }}$. By Lemma 2.5. $\mathrm{X} \cup \mathrm{Y}$ is an isolated point of the set of schemes containing the points and meeting $\Lambda$, and so it satisfies interpolation by [LP16b, Theorem A.7].

3.4. Inductively verifying ind-2. In this subsection, we prove Proposition 3.8, showing ind-2 holds for a given degree $d$, assuming it holds for degree $d-1$. The proof of the Proposition 3.8 is quite analogous to that of Proposition 3.2, in that we specialize all but two of the points to a hyperplane, and then find a scroll of degree $\mathrm{d}$ which is a union of a scroll of degree $\mathrm{d}-1$ inside a hyperplane and a $\mathbb{P}^{k}$, meeting along a $\mathbb{P}^{k-1}$. We now prove Proposition 3.8 assuming Lemma 3.9.

Proposition 3.8. Assuming induction hypothesis ind-2 holds for varieties of degree $\mathrm{d}-1$ with $\mathrm{k}+1 \leq \mathrm{d} \leq 2 \mathrm{k}-2$, it holds for varieties of degree $\mathrm{d}$.

Proof assuming Lemma 3.9 We may assume $k \geq 3$ since the case of $k=1$ is rational normal curves and the case $k=2$ is already covered in [Cos06a, Example, p. 2]. To show $\mathcal{H}_{d, k}^{\text {scroll }}$ satisfies interpolation, we want to show it contains a point corresponding to a variety passing 


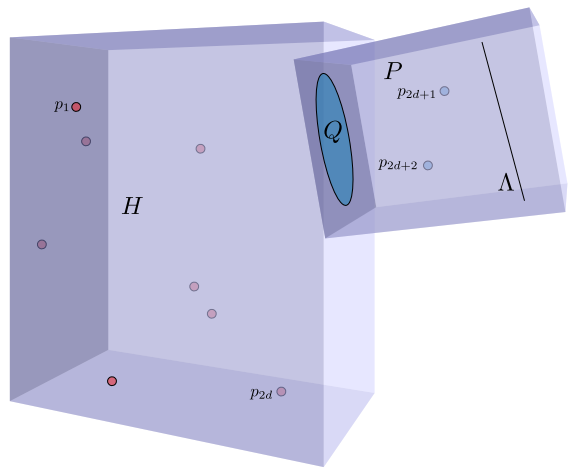

FIGURE 4. A visualization of the idea of the proof of Proposition 3.8, where one inductively specializes $2 \mathrm{~d}$ points to lie in a hyperplane.

through $2 d+2$ general points $p_{1}, \ldots, p_{2 d+2}$ and a general $(2 k-d-$ 1)-plane $\wedge$.

Choose a general hyperplane $H \subset \mathbb{P}^{d+k-1}$ and specialize $\Lambda$ and $p_{1}, \ldots, p_{2 d}$ to be contained in $H$. Let $P$ be the $(2 k-d+1)$-plane spanned by $\Lambda, p_{2 d+1}$ and $p_{2 d+2}$, and let $Q:=P \cap H$. Then, by inductive hypothesis ind-2, there is a scroll of degree $d-1$ and dimension $k$, call it $X$, containing the $2 d$ points $p_{1}, \ldots, p_{2 d}$ and the $2 k-d$ plane Q.

There is a unique $(k-1)$-plane $S$ contained in $X$ and containing $Q$, by Proposition 2.2. Take $Y$ to be the span of $\Lambda, p_{2 d+1}, p_{2 d+2}$, and $S$. The variety $\mathrm{X} \cup \mathrm{Y}$ with reduced scheme structure lies in $\mathcal{H}_{\mathrm{d}, \mathrm{k}}^{\text {broken }} \subset$ $\mathcal{H}_{\mathrm{d}, \mathrm{k}}^{\text {scroll }}$ and contains $\mathrm{p}_{1}, \ldots, \mathrm{p}_{2 \mathrm{~d}+2}$, and $\Lambda$, as desired. Note that the domain and range of the map $\pi_{2}: \Phi \rightarrow\left(\mathbb{P}^{n}\right)^{2 d+2} \times G(2 k-d, n+1)$, as defined in Lemma 3.9. have the same dimension by Lemma 3.9. Furthermore, by Lemma 3.9, when $k>2, \Phi$ has a unique component of maximal dimension, call it $\Phi_{1}$ and possibly one other component of lower dimension, call it $\Phi_{2}$, if it exists. We may therefore choose $\left(p_{1}, \ldots, p_{2 d+2}, \Lambda\right)$ generally to avoid $\pi_{2}\left(\Phi_{2}\right)$.

By a dimension count analogous to Lemma 3.4, there are only finitely many smooth scrolls and finitely many scrolls in $\mathcal{H}_{d, k}^{\text {broken }}$ containing $p_{1}, \ldots, p_{d+2}, \wedge$.

So, applying Lemma 2.5, to the map $\left.\pi_{2}\right|_{\Phi_{1}}$ we see that the point $[\mathrm{X} \cup \mathrm{Y}]$ constructed above is isolated among all schemes containing $p_{1}, \ldots, p_{2 d+2}, \Lambda$. Since $\pi_{2}$ is a map between proper schemes of the 
same dimension and has a point isolated in its fiber, it is surjective, meaning that ind-2 holds for scrolls of degree $d$ and dimension $k$.

To complete the proof of Proposition 3.8, we only need prove Lemma 3.9.

Lemma 3.9. Let $\mathrm{X} \in \mathcal{H}_{\mathrm{d}, \mathrm{k}}^{\text {scroll }}$ and let $\mathrm{F}$ denote the relative Hilbert scheme of $(2 \mathrm{k}-\mathrm{d}-1)$-planes in $\mathcal{V}_{\mathrm{X}}$ over $\mathcal{H}_{\mathrm{X}}$. Define

$$
\Phi=\mathcal{V}_{\mathrm{X}} \times_{\mathcal{H}_{\mathrm{X}}} \cdots \times_{\mathcal{H}_{\mathrm{X}}} \mathcal{V}_{\mathrm{X}} \times_{\mathcal{H}_{\mathrm{X}}} \mathrm{F}
$$

where there are $2 \mathrm{~d}+2$ copies of $\mathcal{V}_{\mathrm{X}}$. Define

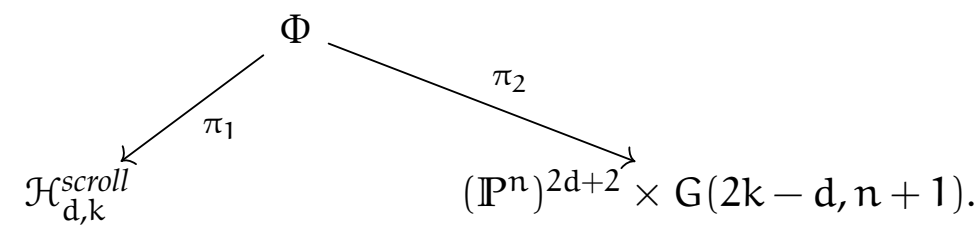

Then, $\Phi$ is irreducible if $2 \mathrm{k}-\mathrm{d}-1 \neq 1$. When $2 \mathrm{k}-\mathrm{d}-1=1$, it has at most two components. If it has two components, one is of dimension $\operatorname{dim}\left(\mathbb{P}^{n}\right)^{2 \mathrm{~d}+2} \times \mathrm{G}(2 \mathrm{k}-\mathrm{d}, \mathrm{n}+1)=(\mathrm{k}+\mathrm{d}-1)(2 \mathrm{~d}+2)+(2 \mathrm{k}-\mathrm{d})(2 \mathrm{~d}-\mathrm{k})$ and the other is of dimension

$$
(k+d-1)(2 d+2)+(2 k-d)(2 d-k)-2(k-2)
$$

Proof. First, the statements regarding irreducibility of $\Phi$ follow immediately from the statements on irreducibility of the Fano scheme as given in Proposition 2.2, coupled with the assumption that $\mathcal{H}_{X}$ and $\mathcal{V}_{\mathrm{X}}$ are irreducible.

Since Proposition 2.2, also gives the dimensions of the irreducible components of $F$, call them $F_{i}$, we have that the dimension of the $i$ th irreducible component of $\Phi$ is $\left(\operatorname{dim} F_{i}-\operatorname{dim} \mathcal{H}_{X}\right)+(2 d+2)\left(\operatorname{dim} \mathcal{V}_{X}-\right.$ $\left.\operatorname{dim} \mathcal{H}_{\mathrm{X}}\right)+\operatorname{dim} \mathcal{H}_{\mathrm{X}}$, as claimed.

3.5. Base case: verifying ind-2 for degree $k$ varieties. We have essentially completed our inductive steps, as pictured in Figure 7. The final step is to examine the base case of interpolation for degree $k$ and dimension $k$ varieties. Recall that smooth degree $k$, dimension $k$ varieties are realized as a Segre embedding $\mathbb{P}^{1} \times \mathbb{P}^{k-1} \rightarrow \mathbb{P}^{2 k-1}$, by Lemma 2.1.

We have two remaining parts of our argument for showing scrolls satisfy interpolation. First, in this subsection, we show that we can find a variety of degree $k$ and dimension $k$ containing $2 k+2$ general points and a general $(k-1)$-plane. 


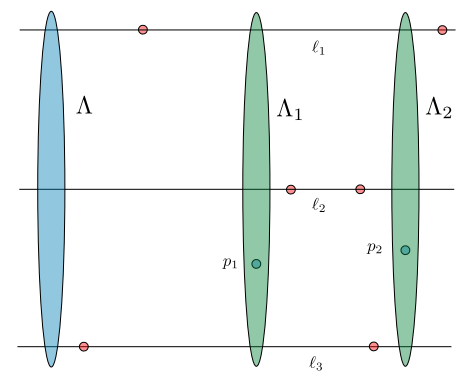

FIGURE 5. A visualization of the idea of the proof of Proposition 3.10, where one specializes pairs of points to lie on lines meeting the given $(k-1)$-plane $\Lambda$.

Then, in subsection 3.6. we show that $\mathcal{H}_{\mathrm{k}, \mathrm{k}}^{\text {scroll }}$ satisfies interpolation, meaning that we can find a scroll in $\mathcal{H}_{\mathrm{k}, \mathrm{k}}^{\mathrm{scroll}}$ through $3 k+3$ general points.

Proposition 3.10. There exist finitely many varieties of minimal degree $S_{1 \mathrm{k}}$ containing $2 \mathrm{k}+2$ general points and a general $(\mathrm{k}-1)$-plane.

Proof. The case $k \leq 2$ is easy so we assume $k \geq 3$. Label the points by $p_{1}, \ldots, p_{2 k+2}$ and the $(k-1)$-plane by $\Lambda$. Define the $k$ lines $\ell_{1}, \ldots, \ell_{k}$, by $\ell_{i}:=\overline{p_{2 i+1}, p_{2 i+2}}$. Specialize the points $p_{1}, \ldots, p_{2 k}$ to general points satisfying the condition that the $k$ lines $\ell_{1}, \ldots, \ell_{k}$ all meet $\Lambda$. We claim that there are only finitely many varieties $S_{1 k}$ which contain such a configuration of points and $\Lambda$.

To see this, note that the ideal of a scroll is generated by quadrics, by [EH87, p. 6]. Since any such $S_{1 k}$ contains three points on each line $\ell_{i}$, namely $p_{2 i+1}, p_{2 i+2}$ and $\ell_{i} \cap \Lambda$, the line $\ell_{i} \subset X$.

Hence, it suffices to show there are a finite, nonzero number of smooth varieties $S_{1 k}$ corresponding to a point in $\mathcal{H}_{k, k}^{\text {scroll }}$ that

(1) contain a $(k-1)$-plane $\Lambda$

(2) contain $k$ lines $\ell_{1}, \ldots, \ell_{k}$, and

(3) contain 2 points $p_{1}, p_{2}$.

Let $X$ be some smooth scroll containing $\Lambda, \ell_{1}, \ldots, \ell_{k}, p_{1}, p_{2}$. By Proposition 2.2, since we are assuming $k \geq 3$, the only $(k-1)$-planes contained in $X$ are the fibers of the projection to $\mathbb{P}^{1}$. Since $\ell_{1}, \ldots, \ell_{k}$ all meet $\Lambda$ at precisely 1 point, they must be lines of the type 1 . Additionally, the two remaining points $p_{1}$ and $p_{2}$ must be contained 


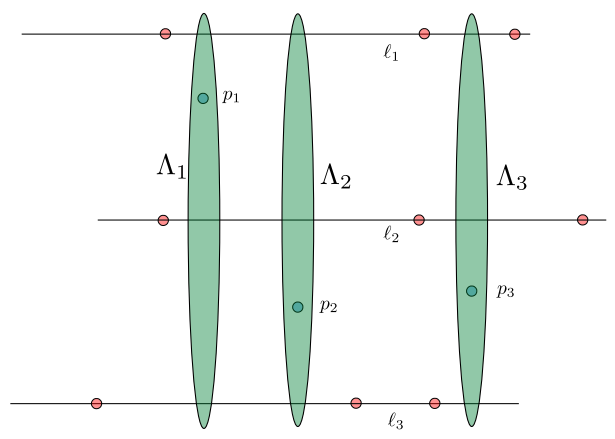

FIGURE 6. A visualization of the idea of the proof of Proposition 3.11, where one specializes triples of points to lie on lines, and then finds a scroll containing those points.

in two $(k-1)$-planes $\Lambda_{1}, \Lambda_{2}$ with $\Lambda_{i} \cap \ell_{j} \neq \varnothing$. By Schubert calculus, there are a positive finite number of planes containing $q_{1}$ which meet $\ell_{1}, \ldots, \ell_{k}$, (In fact, the number of such planes is equal to the number of standard Young tableaux of size $k-1$, although for our argument, we only need that this number is nonzero.) Therefore, there are a positive finite number of choices of pairs of planes $\Lambda_{1}, \Lambda_{2}$ containing $p_{1}, p_{2}$, and meeting lines $\ell_{1}, \ldots, \ell_{k}$.

Now, for any such choice of planes $\Lambda_{1}, \Lambda_{2}$, there is a unique smooth scroll containing $\ell_{1}, \ldots, \ell_{k}, \wedge_{1}, \wedge_{2}, \wedge$ by Lemma 2.6. Hence, in total, there are a finite number of smooth scrolls containing $\Lambda, \ell_{1}, \ldots, \ell_{k}, p_{1}, p_{2}$, as claimed.

3.6. Base case: interpolation for degree $k$ varieties. Before piecing our inductive argument for interpolation of scrolls together, we have to show that $\mathcal{H}_{k, k}^{\text {scroll }}$ satisfies interpolation, which we now do. We do this by constructing an isolated point in a fiber of the map $\pi_{2}$ from (3.2). This fiber has positive dimensional components, and so it takes some care to show that this is indeed an isolated point.

Proposition 3.11. Let $k \geq 2$ and $p_{1}, \ldots, p_{3 k+3}$ be $3 k+3$ general points in $\mathbb{P}^{2 \mathrm{k}-1}$. Then, there is some variety of minimal degree $S_{1^{k}}$ containing $\mathrm{p}_{1}, \ldots, \mathrm{p}_{3 \mathrm{k}+3}$. That is, $\mathcal{H}_{\mathrm{k}, \mathrm{k}}^{\text {scroll }}$ satisfies interpolation. 
Proof. To begin with, specialize the points, as follows: Pick $k$ lines $\ell_{1}, \ldots, \ell_{k}$ so that $\ell_{1}, \ldots, \ell_{k}$ span $\mathbb{P}^{2 k-1}$. Now, specialize the points so that $p_{3 i+1}, p_{3 i+2}, p_{3 i+3}$ are distinct points on $\ell_{i}$ for $1 \leq i \leq k$. This leaves us with 3 general points, which we have labeled as $p_{1}, p_{2}, p_{3}$.

Let $X$ be a smooth scroll of type $\left(1^{k}\right)$ and let $\mathcal{H}^{t+1}\left(\mathcal{V}_{X} / \mathcal{H}_{X}\right)$ denote the relative Hilbert scheme of lines in $\mathcal{V}_{\mathrm{X}}$ over $\mathcal{H}_{\mathrm{X}}$. Define the incidence correspondences

$\Psi:=\mathcal{V}_{\mathrm{X}} \times \mathcal{H}_{\mathrm{X}} \cdots \times \times_{\mathcal{H}_{X}} \mathcal{V}_{\mathrm{X}}$

$\Phi:=\mathcal{H}^{\mathrm{t}+1}\left(\mathcal{V}_{\mathrm{X}} / \mathcal{H}_{\mathrm{X}}\right) \times_{\mathcal{H}_{X}} \cdots \times_{\mathcal{H}_{X}} \mathcal{H}^{\mathrm{t}+1}\left(\mathcal{V}_{\mathrm{X}} / \mathcal{H}_{\mathrm{X}}\right) \times_{\mathcal{H}_{X}} \mathcal{V}_{\mathrm{X}} \times_{\mathcal{H}_{X}} \mathcal{V}_{\mathrm{X}} \times_{\mathcal{H}_{\mathrm{X}}} \mathcal{V}_{\mathrm{X}}$

where $\Psi$ has a product taken over $3 k+3$ copies of $\mathcal{V}_{X}$ and $\Phi$ has a product taken over $k$ copies of $\mathcal{H}^{t+1}\left(\mathcal{V}_{X} / \mathcal{H}_{X}\right)$. Set theoretically (but without specifying a scheme structure) we can describe the closed points of $\Psi$ as

$$
\left\{\left(X, r_{1}, \ldots, r_{3 k+3}\right) \subset \mathcal{H}_{k, k}^{\text {scroll }} \times\left(\mathbb{P}^{2 k-1}\right)^{3 k+3}: r_{i} \in X\right\}
$$

and the closed points of $\Phi$ as

$$
\left\{\left(\mathrm{L}_{1}, \ldots, \mathrm{L}_{\mathrm{k}}, \mathrm{r}_{1}, \mathrm{r}_{2}, \mathrm{r}_{3}, \mathrm{X}\right) \subset(\mathrm{G}(2,2 \mathrm{k}))^{\mathrm{k}} \times\left(\mathbb{P}^{2 \mathrm{k}-1}\right)^{3} \times \mathcal{H}_{\mathrm{k}, \mathrm{k}}^{\text {scroll }}: \mathrm{L}_{\mathrm{i}} \subset \mathrm{X}, \mathrm{r}_{\mathrm{i}} \in \mathrm{X}\right\} .
$$

We have projections
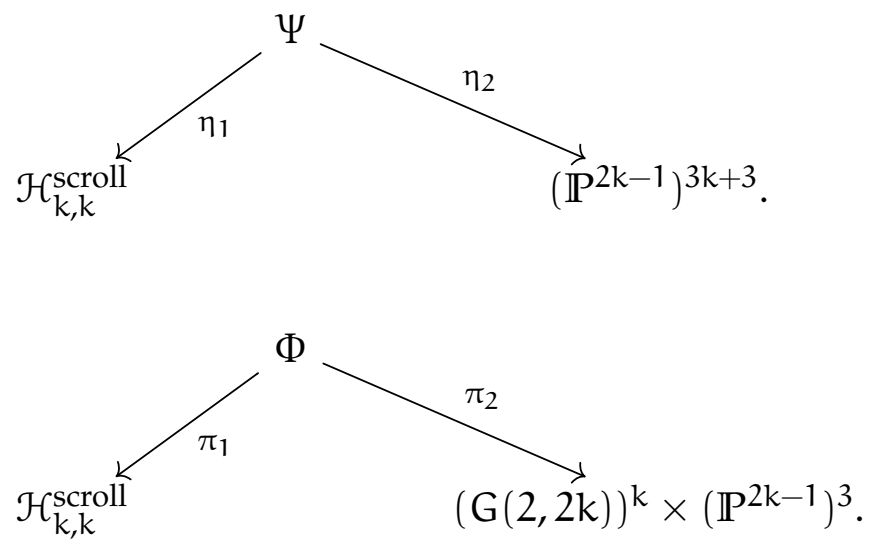

Define $x=\eta_{2}^{-1}\left(p_{1}, \ldots, p_{3 k+3}\right)$ and $y:=\pi_{2}^{-1}\left(\ell_{1}, \ldots, \ell_{k}, p_{1}, p_{2}, p_{3}\right)$. To complete the proof, it suffices to show $X^{2}$ has an isolated point. Note that the locus of $X$ corresponding to smooth scrolls is the same as the locus of $y$ corresponding to smooth scrolls, since any scroll containing $p_{4}, \ldots, p_{3 k+3}$ contains the lines $\ell_{1}, \ldots, \ell_{k}$ as scrolls are generated by quadrics. Therefore, to show $X$ has an isolated point, it 
suffices to show $y$ has an isolated point corresponding to a smooth scroll.

Since we choose $\ell_{1}, \ldots, \ell_{k}, p_{1}, p_{2}, p_{3}$ generally, by Schubert calculus, there are a positive finite number of $(k-1)$-planes containing $p_{i}$ and meeting all lines $\ell_{1}, \ldots, \ell_{k}$. In particular, there is a positive, finite number of three tuples of $(k-1)$-planes $\left(\Lambda_{1}, \Lambda_{2}, \Lambda_{3}\right)$ so that $\Lambda_{i}$ contains $p_{i}$ and meets $\ell_{1}, \ldots, \ell_{k}$. Further, since our parameters were chosen generally, by Lemma 2.6, there is a unique Segre variety $W$ containing any given $\Lambda_{1}, \Lambda_{2}, \Lambda_{3}$ and $\ell_{1}, \ldots, \ell_{k}$.

This corresponds to a point $[W]$ in $y$, hence also some $\left[W^{\prime}\right]$ in $X$. To complete the proof, it remains to show that $\left[W^{\prime}\right]$ is an isolated point in $y$. This follows from Lemma 3.12.

Lemma 3.12. Consider the incidence correspondence $\Phi$ defined in (3.1) with projection maps $\pi_{1}$ to $\mathcal{H}_{\mathrm{k}, \mathrm{k}}^{\text {scroll }}$ and $\pi_{2}$ to $(\mathrm{G}(2,2 \mathrm{k}))^{\mathrm{k}} \times\left(\mathbb{P}^{2 \mathrm{k}-1}\right)^{3}$, as defined in (3.2). Let $\ell_{1}, \ldots, \ell_{k}$ be lines in $\mathbb{P}^{2 k-1}$ and let $p_{1}, p_{2}, p_{3} \in \mathbb{P}^{2 k-1}$ be three points. Define $y:=\pi_{2}^{-1}\left(\ell_{1}, \ldots, \ell_{k}, p_{1}, p_{2}, p_{3}\right)$. Write $\left[W^{\prime}\right] \in y$ as $W^{\prime} \cong \mathbb{P}^{1} \times \mathbb{P}^{k-1}$ via Lemma 2.1. If the lines $\ell_{i}$ are all of type 1 Then, $\left[W^{\prime}\right]$ is an isolated point of $y$.

Proof. We assume $k \geq 3$, as the case $k=2$ holds since $S_{1,1}$ is a quadric hypersurface.

First, by Schubert calculus and Lemma 2.6, there are only finitely many points $[\mathrm{Y}] \in y$ so that all $\ell_{i}$ appear as lines of type 1 . To conclude the proof, it suffices to show that there cannot be a family of scrolls in $y$ containing $W^{\prime}$ so that in the general member of the family, there is some $\ell_{i}$ which appears as a line of type 2. So, suppose for the sake of contradiction, that such a family $f: z \rightarrow C$, for $C \subset y$ and $\left[W^{\prime}\right] \in C$, does exist. We may assume that $C$ is reduced and 1 dimensional (by replacing $C$ with 1 dimensional reduced closed subscheme) and that $C$ is smooth and connected (by replacing $C$ with a connected component of its normalization).

Then, consider the relative Hilbert scheme $\mathcal{H}:=\mathcal{H}^{t+1}(z / C)$ of lines inside $\mathcal{Z}$ over $C$. We claim that $\mathcal{H}$ has two connected components: one whose lines are of type 1 and the other whose lines are of type 2. In particular, this suffices to show such a family over $C$ cannot exist, as such a family would intersect two distinct connected components of $\mathcal{H}$.

To see that $\mathcal{H}$ has the two connected components, we examine the map $f$. Since the fiber of $z$ over a closed point of $C$ is a scroll, we know that the fibers of $\mathrm{g}: \mathcal{H} \rightarrow \mathrm{C}$ over any closed point of $\mathrm{C}$ has 
two smooth connected components of different dimensions, using Proposition 2.2, and our standing assumption that $k>2$.

Now, by Stein factorization, we can factor

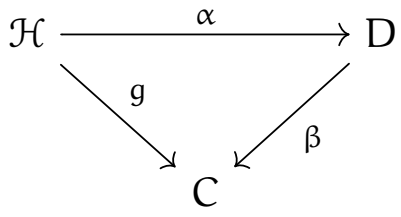

where $\alpha$ has connected fibers, $\beta$ is finite, and D is a normal curve, hence smooth. Further, since $g$ is a dominant map to a smooth curve, we obtain that $\alpha$ is also a dominant map to a smooth curve, hence flat. But, since the connected components of the closed fibers of $\mathrm{g}$ have different dimensions, the connected components of the closed fibers of $\alpha$ have different dimensions, hence different Hilbert polynomials. Since $\alpha$ is flat, D must be disconnected, and hence $\mathcal{H}$ must be disconnected.

3.7. Spelling out the induction. In this subsection, we combine the previous results from this section to prove that smooth scrolls satisfy interpolation. We then conclude that all varieties of minimal degree satisfy interpolation, and hence also strong interpolation in characteristic 0 . See Figure 7 for a visualization of the proof of Theorem 3.13.

Theorem 3.13. If $X$ is a rational normal scroll of minimal degree then $\mathcal{H}_{X}$ satisfies interpolation.

Proof. First, if $X=S_{1 k}$ then this holds by Proposition 3.11 with $j=0$.

Second, suppose $X=S_{2^{t}, 1 k-t}$ with $1 \leq t \leq k-1$. We next show $X$ satisfies interpolation. By Proposition 3.10, inductive hypothesis ind-2 holds for $d=k+1$. By induction, assume it holds for a given degree $d-1$. Then, By Proposition 3.8, it holds for degree $d$, provided $d \leq 2 k-2$. Finally, we obtain that varieties of degree $t+k$ satisfy interpolation by Proposition 3.7.

To complete the proof, it suffices to show that X satisfies interpolation when $d>2 k-1$. We have shown this when $d=2 k-1$. Inductively assume that inductive hypothesis ind-1 holds for degree $d-1$. Then, by Proposition 3.2, it also holds for degree d. Therefore, varieties of degree d satisfy interpolation.

We now prove our main theorem.

Theorem 1.1. Smooth varieties of minimal degree satisfy interpolation. 


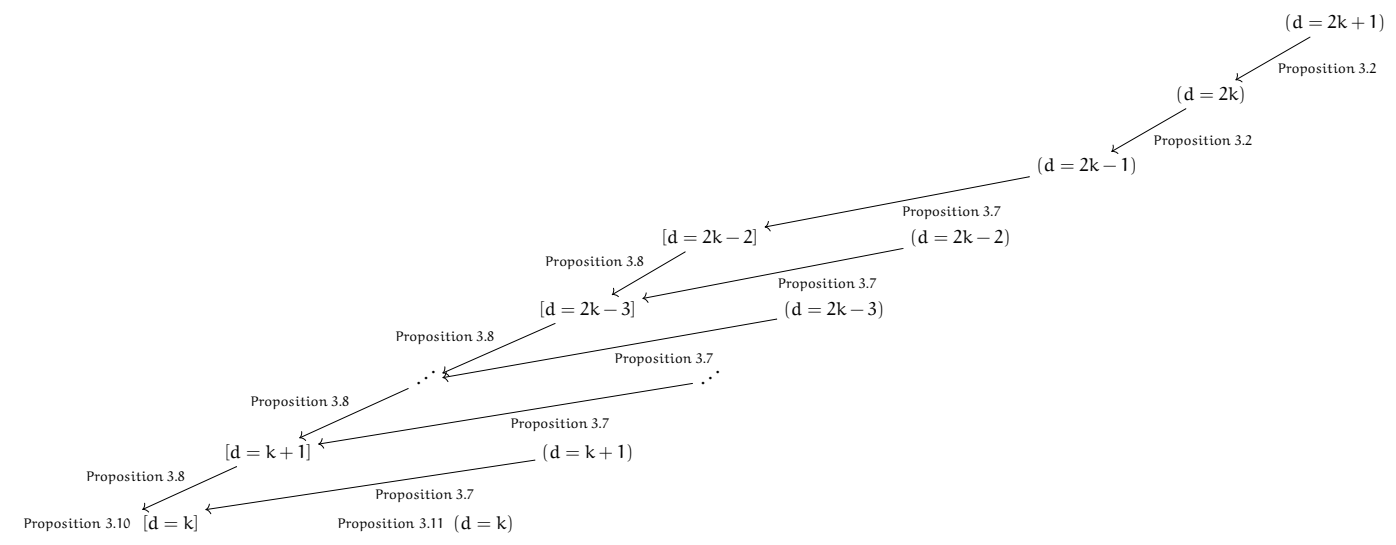

FIGURE 7. This is a schematic diagram for the proof that scrolls satisfy interpolation. The parenthesized expressions $(d=a)$ indicate that scrolls of degree $a$ satisfy interpolation, while the bracketed expressions $[d=a]$ indicate that scrolls of degree a satisfy the hypothesis ind-2. See Theorem 3.13 for a written proof. The arrows point from higher degree to lower degree, and are labeled by the proposition showing that interpolation for the lower degree variety implies interpolation for the higher degree variety.

Proof. First, by [EH87, Theorem 1], we only need show that quadric surfaces, scrolls, and the Veronese embedding $\mathbb{P}^{2} \rightarrow \mathbb{P}^{5}$ satisfy interpolation.

First, quadric surfaces satisfy interpolation because all hypersurfaces do and the 2-Veronese surfaces satisfies interpolation by [LP16b, Theorem 5.6]. Finally, by Theorem 3.13 if $X$ is a scroll then $\mathcal{H}_{X}$ satisfies interpolation.

In order to state an immediate corollary of Theorem 1.1, recall the notion of strong interpolation given in [LP16b, Definition A.5]: An irreducible subscheme of the Hilbert scheme $\mathcal{H}$ parameterizing varieties of dimension $k$ in $\mathbb{P}^{n}$ satisfies strong interpolation if for any collection of planes $\Lambda_{1}, \ldots, \Lambda_{\mathrm{m}}$ of dimensions $\lambda_{1} \geq \cdots \geq \lambda_{\mathrm{m}}$ with $0 \leq \lambda_{i} \leq n-k$ and $\sum_{i=1}^{m} \lambda_{i} \leq \operatorname{dim} \mathcal{H}$, there is some $[Y] \in \mathcal{H}$ meeting all of $\Lambda_{1}, \ldots, \Lambda_{m}$. We say an integral variety $X$ lying on a unique irreducible component of the Hilbert scheme satisfies strong interpolation if $\mathcal{H}_{\mathrm{X}}$ does. 
Corollary 3.14. If $\mathbb{k}$ has characteristic 0 , then smooth varieties of minimal degree over $\mathbb{k}$ satisfy strong interpolation.

Proof. This follows from Theorem 1.1 and the equivalence of interpolation and strong interpolation in characteristic 0 , as follows from the equivalence of conditions (1) and ( $v$ ) from [LP16b. Theorem A.7], using Proposition 2.3.

Remark 3.15 (Strong interpolation in positive characteristic). While Corollary 3.14 shows that varieties of minimal degree satisfy strong interpolation in characteristic 0 , we note that the question of whether varieties of minimal degree satisfy strong interpolation in positive characteristic is still an open problem.

One particularly tantalizing example is that of the 2-Veronese surface in characteristic 2. It is shown in [LP16a, Corollary 7.2.9] that the normal bundle of the 2-Veronese surface does not satisfy interpolation in characteristic 2. (See [LP16b, Definition A.5] for a definition of normal bundle interpolation.) However, it is unknown whether the 2-Veronese surface satisfies strong interpolation in characteristic 2.

\section{REFERENCES}

[ALY15] Atanas Atanasov, Eric Larson, and David Yang. Interpolation for normal bundles of general curves. arXiv preprint arXiv:1509.01724, 2015.

[Bra14] Martin Brandenburg. Tensor categorical foundations of algebraic geometry. arXiv preprint arXiv:1410.1716, 2014.

[Cob] Arthur B. Coble. Associated sets of points. Trans. Amer. Math. Soc., 24(1):1-20.

[Cos06a] Izzet Coskun. Degenerations of surface scrolls and the Gromov-Witten invariants of Grassmannians. J. Algebraic Geom., 15(2):223-284, 2006.

[Cos06b] Izzet Coskun. The enumerative geometry of Del Pezzo surfaces via degenerations. Amer. J. Math., 128(3):751-786, 2006.

[CRV95] MP Cavaliere, ME Rossi, and G Valla. Quadrics through a set of points and their syzygies. Mathematische Zeitschrift, 218(1):25-42, 1995.

[Dol04] Igor V. Dolgachev. On certain families of elliptic curves in projective space. Ann. Mat. Pura Appl. (4), 183(3):317-331, 2004.

[EH87] David Eisenbud and Joe Harris. On varieties of minimal degree (a centennial account). In Algebraic geometry, Bowdoin, 1985 (Brunswick, Maine, 1985), volume 46 of Proc. Sympos. Pure Math., pages 3-13. Amer. Math. Soc., Providence, RI, 1987.

[EP98] David Eisenbud and Sorin Popescu. The projective geometry of the gale transform. arXiv preprint arXiv:math/9807127, 1998.

[LP16a] Aaron Landesman and Anand Patel. Interpolation in algebraic geometry. arXiv preprint arXiv:1605.01117v1, 2016.

[LP16b] Aaron Landesman and Anand Patel. Interpolation problems: Del pezzo surfaces. arXiv preprint arXiv:1601.05840v2, 2016. 
[Ste03] Jan Stevens. Deformations of singularities, volume 1811 of Lecture Notes in Mathematics. Springer-Verlag, Berlin, 2003.

Dept. OF MATHEMATics, StANFord University, Stanford, CA 943052125

E-mail address: aaronlandesman@stanford.edu 